\title{
Immunology in Serbia: from past to present
}

\author{
Ljiljana Sofronic-Milosavljevic • Miodrag Colic
}

Published online: 9 March 2012

(C) Springer Science+Business Media, LLC 2012

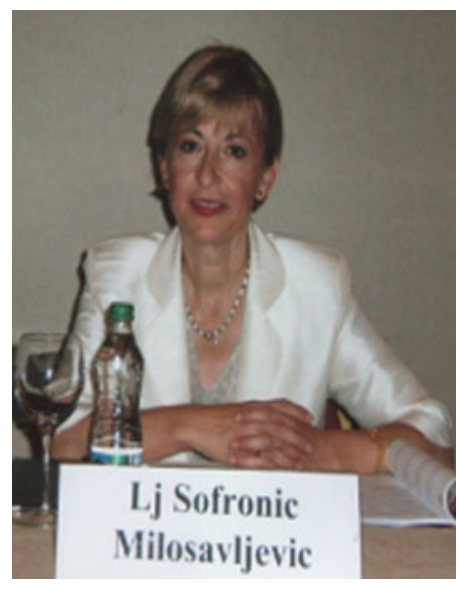

Ljiljana Sofronic-Milosavljevic
The Immunological Society of Serbia (ISoS) originates from the Yugoslav Immunological Society (YIS) founded in 1968 in Belgrade and registered as an individual legal body on September 301974.

As part of YIS, ISoS carried out all the important tasks that the association had set for itself: dissemination of the findings of several groups comprising the core of immunology in Serbia and strengthening cooperation between them; improvement in the teaching of immunology at several universities in Serbia (Belgrade, Novi Sad, Nis); maintaining close cooperation with similar societies inside and between other countries; and finally to serve as a medium through which collaboration between universities and various scientific institutes could be established. By taking steps in relation to the last, ISoS proudly points out that in May 1969, YIS was one of the ten founding members of the International Union of Immunological Societies (IUIS) (http://www.iuisonline.org/iuis/index.php/ about-iuis/history.html) and also contributed to the establishment of the European Federation of Immunological Societies (EFIS) (1975). The central figure from Serbia in this story was Dr. Branislav D. Jankovic. He was among the founders of ISoS and YIS and had a distinguished role in these two associations from the beginning until his

\section{Sofronic-Milosavljevic $(\bowtie)$}

Department for Immunology and Immunoparasitology, Institute for the Application of Nuclear Energy-INEP, University of Belgrade, Banatska 31b, 11080 Belgrade, Serbia

e-mail: sofronic@inep.co.rs

\section{Colic}

Medical Faculty of Military Medical Academy, University of Defense in Belgrade, Crnotravska 17, 11002 Belgrade, Serbia e-mail: mjcolic@eunet.rs retirement. Moreover, he had prominent positions in a number of IUIS (1971-1983) and EFIS bodies (1975-1980). Organization of the first IUIS symposium in Rovinj (Croatia 1971) was the start of a series of specialized international meetings in different areas of immunology that were successfully organized by YIS through the next two decades. This was crowned by organization of the Eighth European Immunology Meeting, which was held in Zagreb from August 30 to September 5 1987. After the breakup of the country (1991), YIS was located in the Federal Republic of Yugoslavia (Serbia and Montenegro) and its work was maintained through ISoS activities. In spite of economic and political upheavals and the civil war, a great achievement at that time was founding the Balkan Association of Immunological Societies, which took place at the 1st Balkan Congress of Immunology BAIS, Belgrade 1995, under the initiative of Dr. Aleksandar Dujic, Dr. Kosta Cuperlovic and Dr. Srdjan Brkic. Since 2006, Serbia has been an independent country and the name of the Society was returned to ISoS. Members of ISoS maintain scientific excellence and organize regular scientific meetings (6-8/year) and national symposia every 3-4 years. Appreciating this, EFIS entrusted the organization of two EFIS/EJI meetings in Belgrade to Dr. Miodrag L. Lukic and ISoS $(2004,2008)$. The current number of ISoS members approaches 80 and is continually expanding with the accession of young scientists. Most of them are involved in basic immunology but some also collaborate in clinical immunology. More information about ISoS and its activities can be found on our Web site http://inep.co.rs/dis/.

The development of immunology in former Yugoslavia, including Serbia, was elegantly described by Dr. Branko Vitale [1] in an invited article in Immunology Today, published on the occasion of the Eighth European Immunology Meeting. 
The history of immunology research in Serbia from the middle of the last century until today could be presented as a survey of the achievements of some of the country's prominent leaders and their research teams. The very beginnings are tied to the name of Dr. Jankovic, who was among the founders of experimental immunology in Serbia during the sixties and a worldwide recognized pioneer in thymus research. Dr. Jankovic introduced the concept that the immune microenvironment is a multisystem composed of components derived from the immune, nervous and endocrine systems. Together with Dr. Katarina Isakovic, he founded the Immunology Research Center at the 'Torlak' Institute of Immunology and Virology in Belgrade, which now bears his name. Their joint effort contributed to knowledge about neuroimmune interactions and neuroendocrinology of the hypothalamo-pituitary-gonadal (HPG) axis. The inspirational setting created by these visionary leaders was prolonged by Dr. Gordana Leposavic (Faculty of Pharmacy, University of Belgrade) and her collaborators, by expanding our understanding of the role of HPG signaling in complex neuroendocrine-immune cross talk, especially concerning programming thymic growth/involution by the perinatal sex steroid milieu and the establishment of immunological sexual dimorphism/diergism.

The era of radiation and cellular immunology in Serbia started with the work of Academician Dr. Miroslav M. Simic in the early sixties at the "Boris Kidric" Institute, Vinca and the "Sinisa Stankovic" Institute for Biological Research (IBISS), University of Belgrade. In the field of radiation immunology, he dealt with immunological restoration of the locally irradiated spleen with circulating lymphocytes. His departure to the Institute of Microbiology and Immunology, School of Medicine, University of Belgrade, resulted in an upswing in research on cellular immunology. As his successors in this field, Dr. Lukic and Dr. Marija Mostarica-Stojkovic studied cellular and genetic determinants responsible for strain differences in susceptibility to experimental autoimmune encephalomyelitis (EAE) induction. The current research of Dr. MostaricaStojkovic and colleagues is focused on the role of mediators produced by antigen-presenting and $\mathrm{T}$ cells in the pathogenesis of autoimmune and chronic inflammatory diseases. This research is based on analysis of gene variants, receptors, cytokines and other related molecules important for initiation, course and therapy response in multiple sclerosis (MS) and EAE, as well as in psoriasis and chronic graft rejection.

At IBISS investigations in the field of cellular immunology were passed to Dr. Stanislava Stosic-Grujicic who, with her collaborator Dr. Lota Ejdus-Konstantinovic, expanded research on the role of macrophages in T-cell activation. During the last 20 years, this group explored functioning of the immune system in physiological and pathological conditions with special focus on cytokine networking and regulation. The investigations comprised basic and clinical aspects of autoimmunity, inflammation, infection, malignancy and hematopoiesis in animals and humans. A brand new field, opened by Dr. Milena Kataranovski at IBISS in 2006, covers the influence of the environment and/or infection on the host immune response.

Investigations on the role of the thymus, initiated by Dr. Jankovic, were actively continued by Academician Dr. Miodrag Colic, Dr. Dujic and colleagues from the Institute for Medical Research at the Military Medical Academy, Belgrade, some 30 years ago. They shed light on the role of various types of trauma, hormones and drugs on the histomorphology of the thymus and cell populations inside it. At the same time, they dealt with the immune response after injury and some aspects of clinical immunology: transplantation and HIV infection. In the last two decades, the focus was on immunobiological characteristics of dendritic cells, immunoregulation in chronic inflammation processes and wound healing.

Studies on radiation immunobiology were also started in the field of parasitology by Dr. Cuperlovic and Dr. Miodrag Movsesijan at the Institute for the Application of Nuclear Energy-INEP, University of Belgrade. They demonstrated that irradiated parasites can successfully be used as vaccines. As a link between the past and the future, parasites retain a central place in the research, which, in the last decade, was focused on the capacity of helminths to modulate the immune response of the host to irrelevant antigens including autoantigens (Dr. Ljiljana SofronicMilosavljevic).

The names of Dr. Vladislava Nikolic and Dr. Vojislav D Miletic are related to the establishment of immunochemistry in Serbia (during the seventies, previous century) at the Institute for Medical Research, IMI, University of Belgrade and at the Blood Transfusion Institute, Belgrade, respectively. Complement-immunoglobulin interactions were in the focus of Dr. Miletic research. Work of Dr. Nikolic, together with Dr. Marko Vukotic, on architecture of the immunoglobulin molecules and their functional characteristics represented the base for further extensive study of collaborators from their research team (Dr. Nadezda Milosevic-Jovcic, Dr. Vesna Ilic). Cellular and molecular mechanisms involved in the regulation of hematopoesis in normal and pathological conditions were also studied successfully at this Institute (Dr. Diana Bugarski, Dr. Gordana Jovcic).

At the beginning of the eighties, Academician Dr. Ivan Spuzic initiated research in the field of tumor immunology in Serbia, at the Institute for Oncology and Radiology in Belgrade. This is still concentrated on the effects of immunomodulatory agents on the phenotype and functional characteristics of immune cells: lymphocytes, 
monocytes, NK cells in malignancies (Dr. Gordana Konjevic, Dr. Zorica Juranic).

The first scientific research project in immunology at the School of Medicine, University of Kragujevac began in 1995. Over the past 15 years, investigations comprised effector and immunomodulatory mechanisms in some autoimmune diseases and malignancies (Dr. Nebojsa Arsenijevic, Dr. Snezana Zivancevic-Simonovic). Dr Lukic joined this research team in 2005, enabling a breakthrough in the investigations of immune phenomena at the molecular level and studies on immunoregulation and pathogenesis of autoimmune diseases.

It is also worth mentioning that there were some research projects, mostly related to clinical immunology that were conducted either in Belgrade (Dr. Mirko Mikuska, Institute for Health Protection of Mother and Child "Dr. Vukan Cupic", Belgrade) or at Schools of Medicine in two other University centers in Serbia: Novi Sad and Nis.

In conclusion, the Immunological Society of Serbia survived through difficult times and proved the quality of its work through publications in renowned international journals. Regarding future perspectives, Serbian immunologists hope that their explorations will be in step with those performed by the European and international immunological communities. Finally, we consider our presentation of the Society in this publication to be a great honor and recognition.

\section{Research highlights}

This special issue of the journal Immunologic Research provides an insight into the themes that preoccupy the attention of immunologists in Srbia. We have assembled articles of selected members of ISoS that altogether represent the teamwork realized through the past and present national projects. Many of them summarize decades of research and multigenerational continuity in certain fields.

There is a growing body of evidence indicating an important role of the neonatal steroid milieu in programming sexually diergic changes in thymopoietic efficiency, which leads to phenotypic and functional remodeling of the peripheral T-cell compartment. Leposavic et al., provided novel evidence of the role of the neonatal sex steroid milieu in programming rat thymic growth and involution, as well as sexual diergism in thymopoiesis. This research group contributed to an understanding of the putative role of changes in catecholamine-mediated communications in the thymopoietic alterations in adult neonatally androgenized rats.

Dendritic cells (DCs) are considered as key antigenpresenting cells, and owing to their capacity to regulate immunity and tolerance, these cells are attractive candidates to be used as vaccines in the broad field of immunotherapy, ranging from chronic inflammatory/ autoimmune diseases to cancer. In the review of Dzopalic et al., authors summarize current data related to the effect of individual and joint pattern recognition receptor (PRR) agonists on DCs and Langerhans-like cells derived from monocytes. They demonstrate that co-stimulation of DCs with different PRR ligands induced superior T helper (Th)1 and Th17 immune responses, compared to effects of single agonists. In addition, nanoparticles applied as carriers for PRR agonists stimulated much better the immune response compared to the agonists without carriers. These findings may be helpful as a new strategy to improve tumor immunotherapy.

The involvement of the immune system, especially $\mathrm{T}$ cells, in regulation of hematopoiesis was suggested a long time ago. More recently, the link between interleukin (IL)17 and hematopoiesis, through stimulation of granulopoiesis and neutrophil trafficking, has been recognized. Data reviewed by Krstic et al., showed that IL-17 also affects other cells of the hematopoietic system, such as erythroid progenitors and mesenchymal stem cells. They confirmed that IL-17 is an important component of adaptive immunity and part of a regulatory cytokine network involved in hematopoietic regulatory mechanisms, both during steady state and altered hematopoiesis.

Etiology and pathogenesis of any disease rise upon interplay between genetic and environmental factors. New technologies identified genetic polymorphisms associated with disease susceptibility among which immunologically relevant genes are significantly overrepresented, and they might be used as biomarkers to identify accurate diagnosis, predict clinical disease course and response to therapy. In this issue, the review by Pravica and colleagues focuses on the recent progress in research on MS genetics with special emphasis on the possibility to use single nucleotide polymorphism of candidate genes as biomarkers of susceptibility to this chronic inflammatory, demyelinating and neurodegenerative disease of the central nervous system (CNS), as well as the response to therapy.

Experimental autoimmune encephalomyelitis shares many clinical, histological, immunological and genetic features with human demyelinating diseases, including MS, and is, therefore, widely used to gain an important insight into MS pathogenesis and to validate new targets for MS therapy. One approach is comparative investigation of strains of rats, such as Dark Agouti (DA) and Albino Oxford (AO) rats that are at the opposite poles of susceptibility to EAE induction. AO strain is highly resistant to EAE induction, while DA strain is highly susceptible. Momcilovic et al. postulated that CNS intrinsic regulatory mechanisms might contribute to the resistance of $\mathrm{AO}$ rats 
to the induction of EAE. They observed that the expression of chemokine CXCL12 was significantly downregulated in DA rats at the peak of EAE and strongly upregulated in AO rats analyzed at the same time point. It appears that CXCL12 may largely contribute to the resistance of animals to EAE induction, and it is tempting to speculate that it might be protective in MS pathogenesis, as well.

Although the different types of immune cells and molecular mechanisms are involved in the pathogenesis of chronic inflammatory diseases, such as MS/EAE, the research of Dimitrijevic and the colleagues has mainly been focused on recent findings related to the role of mediators forming the major interface between the brain and immune system in establishing thymic central tolerance and macrophage functions, strongly indicating the significance of alterations in the neuron-immune interface for the development of inflammatory autoimmune diseases.

Cellular and molecular mechanisms of the pathogenesis of immunoinflammatory diseases like type 1 diabetes are intensively studied by Stojanovic et al. who provided an overview of the biological properties of macrophage migration inhibitory factor as a molecule with versatile functions. They emphasized its relevance to normal physiological function of endocrine pancreas, for example, insulin secretion and to protection of beta cells from infectious agents while, after an insult, it becomes an autodestructive molecule that can harm beta cell by provoking their apoptosis or necrosis or inducing their immortality.

Important contribution to the investigations of immunopathology in inflammation/autoimmunity is provided by two papers from the same group. The first paper prepared by Milovanovic et al. is focused on IL-33/ST2 axis and its role in T-cell-mediated autoimmune disorders and in antitumor immunity. Beside evidence that ST2 deletion and exclusion of IL-33/ST2 axis is accompanied with enhanced susceptibility to dominantly T-cell-mediated organ-specific autoimmune diseases (in experimental model of multiple low dose streptozotocin-induced diabetes -MLD-STZ and EAE), they showed that ST2 deletion/ blocking may enhance antitumor immunity and stimulate NK cell activity.

The second paper prepared by Radosavljevic et al. summarizes the knowledge regarding the role of Galectin-3 in T-cell-mediated inflammatory (auto) immunity (disease models of human pathology used in the study included: Con A-induced hepatitis, MLD-STZ diabetes and EAE) and tumor rejection (in B16-F1 malignant melanoma model). The authors suggest that selective inhibition of Galectin-3 may be a useful therapeutic approach in the treatment of autoimmune and malignant diseases.

Quite a different approach to the potential treatment of autoimmune disorders is presented in this issue by the review of Ilic et al. The authors point out the ability of
Trichinella spiralis-stimulated DCs to polarize the immune response toward Th2 and regulatory mode in vitro and in vivo and also the capacity of this parasite to modulate EAE as a model of autoimmune disease. Their data provide further evidence that the helminth parasite $T$. spiralis possesses the capacity to retune the immune cell repertoire, acting as a moderator of the host response not only to itself but also to third party antigens.

The influence of environmental factors, such as infection with Aspergillus fumigatus, on the host immune response is presented in the review given by Mirkov et al. The authors point out the importance of the use of murine models of systemic aspergillosis in exploring several aspects of the disease and host response, both innate and adaptive. They demonstrate the relevance of the inflammatory milieu for clearance of nonlethal Aspergillus infection from the spleen, as a site of initial conidia accumulation, as well as other peripheral tissues affected. The study of various aspects of systemic aspergillosis could provide a valuable contribution to the development of antifungal drugs.

Skin is the site of many immune responses reactive to different stress agents like microbes, foreign substances or trauma. Two papers in this issue deal with innate and/or adaptive immune responses in the skin. Popov et al. reviews data in the literature related to rat contact hypersensitivity as a T-cell-mediated skin inflammatory reaction to cutaneous exposure to small sensitizing chemicals, haptens. They present results concerning the importance of genetic background both in the induction as well as in the expression of reaction to dinitrochlorobenzene and discuss them in terms of inflammatory and immune mechanisms of both sensitization and elicitation phases.

Thermal injury, as a form of severe trauma, induces simultaneous pro- and anti-inflammatory response. There are many published data that show evidence on the increased accumulation of granulocytes in the lungs or liver during thermal injury. On the other hand, the review of Draskovic-Pavlovic et al., presented in this issue, summarizes results that demonstrate reduced granulocyte influx in the wound that heals in conditions of thermal injury. The authors also provide evidence that indicates impaired signal transduction in granulocytes following thermal injury, as well as their divergent response regarding adhesiveness, oxidative burst and nitric oxide production at the wound site.

Tumor immunology as attractive, actual and inspiring field for investigation is represented by two papers in this issue. The research is focused on particular components of the immune response to tumors, clinical-stage relation to these components and possible therapeutic approaches. Paper by Konjevic and colleagues deals with the role of NK cells in cancer, showing clinical-stage dependent 
impairment of NK cell activity in breast cancer, Hodgkin's disease and non-Hodgkin's lymphoma. Long-term immunomonitoring of patients with malignancies revealed the kinetics of NK cell modulation during chemoimmunotherapy. The authors present data on NK function and novel families of NK receptor expression in healthy individuals that may be of help in NK cell profiling. Moreover, this review gives novel aspects of NK cell activity modulation by cytokines approved for immunotherapy, such as IFN and IL-2, used for the treatment of melanoma and other malignancies.

The review of Maksimovic Ivanic et al. described Tumor Necrosis Factor Related Apoptosis Inducing Ligand (TRAIL), as the most selective natural mediator of innate immunity in cancer cell death. However, the resistance to TRAIL-mediated apoptosis was recognized together with numerous molecules responsible for that. This paper reviews the possible routes of reconstitution of sensitivity to TRAIL-mediated immune response by specific modulation of different signals responsible for the development of resistance. The authors contributed to this scientific problem by investigating the effects of antiretroviral drug-saquinavir, chemically modified by covalent attachment of NO (Saq-NO). Results indicated that SaqNO, as a brand new compound, exhibited enhanced antitumor activity, retained the antiviral property and also showed complete lack of toxicity.

We hope that the reviews in this issue will attract attention of international scientific community and promote new collaborations with Serbian immunologists, members of ISoS.

\section{Reference}

1. Vitale B. The development of immunology in Yugoslavia. Immunol Today. 1987;8:163-7. 